\title{
Photogrammetric analysis of additive manufactured metallic open cell porous structures
}

\author{
Samuel Evans, Eric Fones, Peter Fox and Chris Sutcliffe \\ The School of Engineering, The University of Liverpool, Liverpool, UK
}

\begin{abstract}
Purpose - This paper aims to introduce a novel method for the analysis of open cell porous components fabricated by laser-based powder bed metal additive manufacturing (AM) for the purpose of quality control. This method uses photogrammetric analysis, the extraction of geometric information from an image through the use of algorithms. By applying this technique to porous AM components, a rapid, low-cost inspection of geometric properties such as material thickness and pore size is achieved. Such measurements take on greater importance, as the production of porous additive manufactured orthopaedic devices increases in number, causing other, slower and more expensive methods of analysis to become impractical.

Design/methodology/approach - Here the development of the photogrammetric method is discussed and compared to standard techniques including scanning electron microscopy, micro computed tomography scanning and the recently developed focus variation (FV) imaging. The system is also validated against test graticules and simple wire geometries of known size, prior to the more complex orthopaedic structures.

Findings - The photogrammetric method shows an ability to analyse the variability in build fidelity of AM porous structures for use in inspection purposes to compare component properties. While measured values for material thickness and pore size differed from those of other techniques, the new photogrammetric technique demonstrated a low deviation when repeating measurements, and was able to analyse components at a much faster rate and lower cost than the competing systems, with less requirement for specific expertise or training.

Originality/value - The advantages demonstrated by the image-based technique described indicate the system to be suitable for implementation as a means of in-line process control for quality and inspection applications, particularly for high-volume production where existing methods would be impractical.
\end{abstract}

Keywords Laser forming

Paper type Research paper

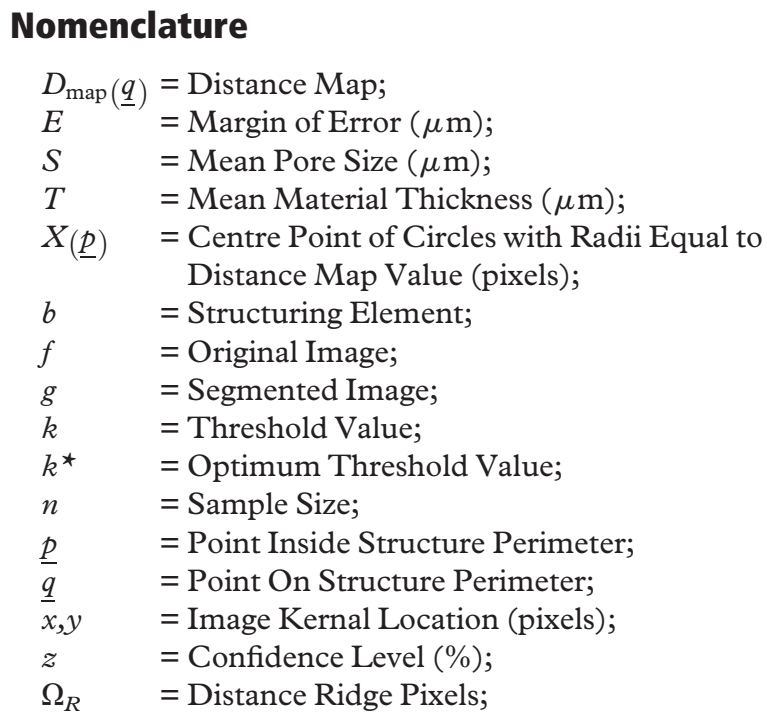

The current issue and full text archive of this journal is available on Emerald Insight at: www.emeraldinsight.com/1355-2546.htm

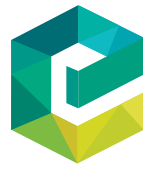

Rapid Prototyping Journal

24/8 (2018) 1380-1391

Emerald Publishing Limited [ISSN 1355-2546]

[DOI 10.1108/RPJ-05-2017-0082]

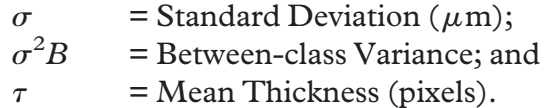

\section{Introduction}

Amongst other applications, open cell porous coatings are used in orthopaedic implant design to improve fixation, and have replaced cement and bone screws as the favoured method of attaching implants to existing bone (Friedman et al., 1993). The use of porous structures allows for bone in-growth to improve security, increasing the lifetime of the device (Bobyn et al., 1999; Jeyapalina et al., 2014). Porous structures can be fabricated using a number of methods, such as plasma spraying and sintering. These methods, however, do not produce

\footnotetext{
(C) Samuel Evans, Eric Jones, Peter Fox, Chris Sutcliffe. Published by Emerald Publishing Limited. This article is published under the Creative Commons Attribution (CC BY 4.0) licence. Anyone may reproduce, distribute, translate and create derivative works of this article (for both commercial and non-commercial purposes), subject to full attribution to the original publication and authors. The full terms of this licence may be seen at http://creativecommons.org/licences/by/4.0/legalcode
}

Received 8 May 2017

Revised 26 October 2017

23 February 2018

12 March 2018

Accepted 16 March 2018 
repeatable structures with precisely controlled properties, thereby limiting meaningful comparative analysis of the porous material and inhibiting the quantifying of variability in the manufacturing process for quality assurance (Ryan et al., 2006). With porous structures requiring specific properties to maximise functionality, understanding this variability is key to producing consistent and reliable components.

Metallic additive manufacturing (AM) is a technology that allows for near identical porous structures to be designed and created indefinitely, with high repeatability and control over the properties required (including porosity, pore size and anisotropy of the material). This comes as a consequence of creating the structure directly from CAD and modifying the unit cell-based structure by way of bespoke algorithms to maximise the strength of both the component and the in-grown bone (Mullen et al., 2010; Mullen et al., 2009). When analysing components with repeatable structure designs, variation in material thickness and pore size can be analysed through comparison of measurements made to previously held data. As all parts manufactured are identical in design and should therefore be identical in production, any difference in measurements will come as a direct result of uncontrolled changes in the AM process. This capability to highlight process-induced errors is unique to AM porous structures and lends itself to non-destructive quality and process control where high volumes of components are required to be inspected. This is in contrast to traditional methods of manufacturing porous components that do not permit such actions without incurring excessive cost and time penalties.

Three non-destructive measurement techniques, which leave porous structures intact post-analysis, are currently applied in industry. These are scanning electron microscopy (SEM), focus variation (FV) and micro computed tomography ( $\mu$-CT), with each technique presenting advantages and disadvantages that satisfy different analysis requirements. SEM analysis produces values for both pore size and strut diameter of the porous material through manual measurements of a calibrated image captured using the microscope (Mullen et al., 2009; Tomlins et al., 2004). This process takes several minutes per image, in addition to time required for sample preparation and vacuuming of the chamber for imaging. As a result of the manual method, the technique exhibits significant potential for error, and suffers from a lack of automation. Only a small sampling area, typically several millimetres squared at most, is used due to the high magnification $(25 \times)$ when capturing images (Muth et al., 2013).

Focus variation methods operate by vertically sweeping the focus plane of a lens (with a shallow depth of field) to capture slices of a component across a range of heights, at a vertical resolution of $10 \mathrm{~nm}$, and stitches them together to create threedimensional (3D) topographical data (Leach, 2011; Danzl et al., 2011). The FV system is able to capture data rapidly from the surface of a component at a resolution of $2 \mu \mathrm{m}$; however, this method allows for only a small number of struts to be measured at a time following imaging, making the complete analysis of a structure laborious and time consuming, and preventing analysis of pore size, limiting practical use for in-line component inspection (Leach, 2011; Danzl et al., 2011). In contrast with other methods, $\mu-\mathrm{CT}$ is able analyse a $3 \mathrm{D}$ porous structure internally. Scanning X-rays through a part allows for a $3 \mathrm{D}$ model to be generated that can be used to measure pore size, porosity, permeability, build accuracy and the surface roughness of individual struts (Yue, 2011). $\mu$-CT exhibits a high accuracy with a resolution up to $1 \mu \mathrm{m}$ dependent on the voxel size used to generate the $3 \mathrm{D}$ model, a product of the volume and density of the scanned component. In metallic porous material, scan resolution has been practically demonstrated down to $9 \mu \mathrm{m}$ (Yue, 2011). The process of acquiring $\mu$-CT data, however, can take several hours and cost hundreds of pounds per sample analysed. The method is also computationally demanding, requiring specialised post processing of the acquired data to quantify porous structures (Yue, 2011). $\mu$-CT is therefore suitable for spot checks or development of porous material but not high-volume in-line inspection.

This paper introduces an analysis technique to address limitations in the existing methods. Most critical drawbacks of current systems include measurement time, complexity of operation and cost. By overcoming these, a practical method suitable for high-volume inspection is introduced. The proposed system adopts an off-the-shelf DSLR camera and open source image analysis software, FIJI (LOCI, US). Existing analysis algorithms are applied to images of porous components to quantify material thickness and pore size in a rapid fashion. Unlike existing systems, the image analysis method is capable of measuring both material thickness and pore size properties of a porous structure algorithmically from a single image. Automation and refinement of the image processing were also possible through the use of the Python programming language to improve the suitability of the method for high-volume manufacturing applications. With a rapid, lowcost system, a 100 per cent inspection regime, even that containing large numbers of components, may be performed on porous components as part of quality control and assurance. While not very widely used currently, similar systems have been applied to validation of simpler AM-produced porous structures to analyse disparity between vertical and horizontal struts and compare values to the ideal CAD model (Vanderesse et al., 2016). This existing study demonstrated the capability of image analysis methodologies to quantify geometric variation between lattices built under different conditions and highlighted local thickness measurements as a viable method of determining material width. However, measurements were limited to regular lattices and therefore were not applicable to randomised osseointegrating surfaces.

\section{Materials and methods}

Porous test specimens were produced from Commercially Pure Grade 1 Titanium (CpTi) powder (Sumitomo Corporation, JP) with a mean particle diameter between 15 and $45 \mu \mathrm{m}$ (suitable for laser-based AM production). This material was selected for being representative of that used in commercially available porous orthopaedic implants. Manufacturing was performed using an MCP Realizer 250 SLM system (MCP Tooling Technologies, GB), a Laser Based Powder Bed Fusion (LBPBF) machine using a $50 \mu \mathrm{m}$ layer thickness. Built samples consisted of porous material surrounded by a solid wall, with a solid square placed in one corner of the material (fiducial marker) for consistent orientation when capturing images, as shown in Figure 1. At 
Figure 1 Porous test specimen
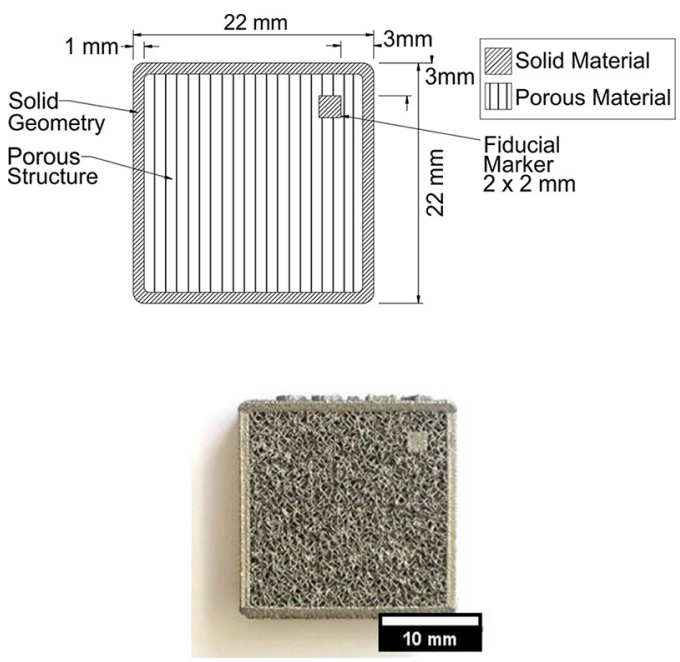

solid-porous interfaces an overlap of $200 \mu \mathrm{m}$ was included ensuring a bond between the two material types during manufacturing, improving durability.

The open celled structure comprised individual, interconnected struts created from the 30 per cent pseudorandomisation of octahedral shapes (Robinson, 2014; Mullen et al., 2010; Mullen et al., 2009). The randomised porous structure, intended to mimic the architecture of human trabecula bone, was generated by using a random seed that controlled the distance by which the nodes at the corners and centre of the octahedral cells were moved (Robinson, 2014). This allowed for identical randomisation to be applied every time the porous material was generated. By using the same seed on an identical starting component, duplicate porous CAD models were produced. Using different random seeds to generate the structure produced similar designs, but containing alternative strut locations. A key advantage of this repeatable manufacturing method for quality control purposes is the possibility to compare built components with one another to determine the fidelity of the structures and to track variation in manufactured parts for continuous process control.

Following generation and randomisation, each strut was converted to slices equivalent to the thickness of the powder layer, and used to produce a point cloud. At points where strut vectors intersected the layer being manufactured, the laser spot was directed and a melt pool created which rapidly solidified. Struts were formed from six pools of material, dependent on the dimensions of the unit cell used in generating porous material designs, fused together at the required angle and spacing as dictated by the machine input data. Values for spot size of the Ytterbium Fibre Laser's Gaussian beam profile used in the manufacturing process (defined as the area in which 86 per cent of the beams energy lies measured between points of $1 /$ $e^{2}$ of the peak intensity value), power level of the beam at the powder bed during manufacturing, and the exposure time for each point in the porous structure were optimised to create fully dense material following solidification of the melt pools (Steen et al., 2010).
Once built, specimens were removed from the build plate, and cleaned using distilled water in a Kerry ultrasonic bath (Guyson, GB) for $30 \mathrm{~min}$. Images of the surface of the porous specimens were captured using a Canon 70D DSLR camera and Macro EF $100 \mathrm{~mm}$ lens (Canon Inc., Japan). The camera and porous specimens were mounted on a jig, ensuring images were captured in a repeatable fashion. Specimens were lit using consistent ambient illumination. Figure 2 shows the manufactured imaging jig and key dimensions used in the study. Dimensions were selected to align test specimens with the central axis of the lens and match the height of the camera above the specimen to the minimum focusing distance to maximise magnification while retaining a sharp image, producing a pixel resolution of $8 \mu \mathrm{m}$.

Camera settings were selected for use in close-up image capturing, ensuring bright, uniformly lit struts and high contrast between struts and pores within the images, selected following a pilot study and testing with the image binarisation methodology described below. Specific settings were set as: an ISO speed of $1,000 \mathrm{~ms}$, a shutter speed of $6,000 \mathrm{~ms}$ and an aperture value of 16 (Canon Inc., 2015).

\section{Initial image processing}

Images were captured in RAW format using the timer mode to prevent unwanted vibration, and converted to the TIFF file format using Digital Photo Professional (Canon Inc., Japan). This was followed by conversion to 8-bit greyscale and thresholding via Otsu Binarisation, displaying struts as white and pores as black in resulting images, both carried out in Python OpenCV (Schindelin et al., 2012; Gonzalez et al., 2008). The thresholding methodology analyses the histogram of an image to select a suitable grey value to divide the image into the black background (representing pores) and the white subject, i.e. the solid material (Schindelin et al., 2012; Gonzalez et al., 2008). This is performed by altering the 8-bit value of the pixel dependent on how light or dark it is. In the case of this study, Otsu Binarisation was applied to determine the optimum threshold value using equation (1):

$$
\sigma_{B}^{2}\left(k^{*}\right)=\max _{0 \leq k \leq L-1} \sigma_{B}^{2}(k)
$$

Equation (1) evaluates different values of $k$, the threshold value, to reduce $\sigma_{B}^{2}$, the between-class variance, to its

Figure 2 (a) Schematic of a jig and camera used in capturing images of porous components and (b) the manufactured jig and camera

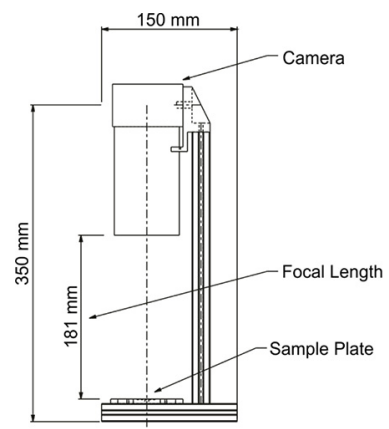

(a)

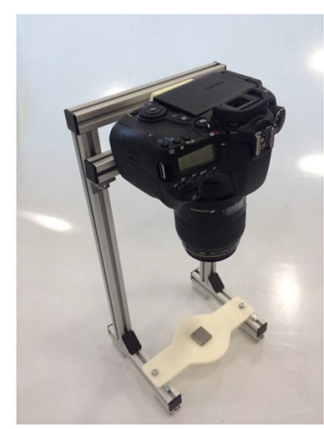

(b) 
minimum. This point indicates where the optimum threshold, $k^{\star}$, of the image lies between 0 and 255 for an 8-bit greyscale image. Once selected, the threshold value is applied to equation (2) to determine which pixels become black (below threshold value) and which become white (above threshold value) (Gonzalez et al., 2008):

$$
g(x, y)=\left\{\begin{array}{l}
1 \text { if } f(x, y)>k^{*} \\
0 \text { if } f(x, y) \leq k^{*}
\end{array}\right.
$$

Following thresholding, opening and closing operations were used to filter small defects from the image, also performed in Python OpenCV (Gonzalez et al., 2008). The opening and closing operation applies dilation (a process in which pixels are added to the outer perimeter of white areas of a binary image) and erosion (a process in which pixels are removed from the perimeter) to remove small areas of noise from the image. Applying an opening filter requires the application of erosion, followed by an equal amount of dilation, using a structuring element, $b$, to determine where pixels were added to or removed from the surface of features, denoted by equation (3) (Gonzalez et al., 2008):

$$
f \bullet b=(f \ominus b) \oplus b
$$

Similarly a closing filter applies the same procedure but with opposite operations i.e. dilation is performed first, followed by equal erosion as in equation (4) (Gonzalez et al., 2008):

$$
f \bullet b=(f \oplus b) \ominus b
$$

During processing, images were eroded and dilated by five pixels for both opening and closing operations. An image processed with the described method is shown in Figure 3.

Processed images became a basis from which two geometric features were extracted, material thickness and pore size. Material thickness measured the width of the solid phase of the porous structure (struts and nodes) to quantify the average value across the material. Pore size analysed the width of visible pores, between the struts and nodes, to determine the mean visible pore size of the structure. Measurements were made from the captured images with the aim of being applied to tracking variation in the manufacturing process and to allow for go/no go inspection and quality control.

\section{Material thickness analysis}

The first method of analysis used local thickness measurements to determine the mean material thickness of the porous structure. This differed from the strut diameter measurements made by other existing methods in that it measured all parts of the material comprising the porous structure, not just the connecting struts. An initial distance map of the image was produced in which a value was assigned to each pixel equal to the distance from that pixel to a black pixel with grey scale value of 0 in Euclidian space (Dougherty and Kunzelmann, 2007; Hildebrand and Rüegsegger, 1997). Following this was the generation of a distance ridge formed from the highest values in the distance map. This located the centreline of solid structures formed from white pixels. For each pixel in the distance ridge, a circle was centred with a circumference equal to the distance map value, corresponding to the diameter of the material at that point along the ridge (Dougherty and Kunzelmann, 2007). The local thickness, $\tau$, is represented in equation (5) where, $q$ is a set of points on the perimeter of a feature, $p$ is a set of points within the perimeter and $D_{\text {map }}$ is the distance map of the image (Dougherty and Kunzelmann, 2007):

$$
\tau(\underline{p})=2 \cdot \max _{\underline{q} \in X(\underline{p})}\left(D_{\operatorname{map}}(\underline{q})\right)
$$

The set $\widetilde{X}_{(\underline{p})}$, shown in equation (6), represents the centre points of all circles with radii equal to the value of the distance map at that point, contained within the set $\Omega_{R}$, the pixels in the distance ridge of the structure, and including point $\underline{p}$ (Hildebrand and Rüegsegger, 1997):

$$
\tilde{X}(\underline{p})=\left\{\underline{x} \in \Omega_{R} \mid \underline{p} \in \operatorname{sph}\left(\underline{x}, D_{\operatorname{map}}(\underline{x})\right)\right\}
$$

From this analysis, an image showing the placement and diameter of fitted circles (represented by a colour range with

Figure 3 (a) A captured image of a porous sample prior to processing and (b) image following cropping, conversion to 8-bit, Otsu Binarisation and filtering

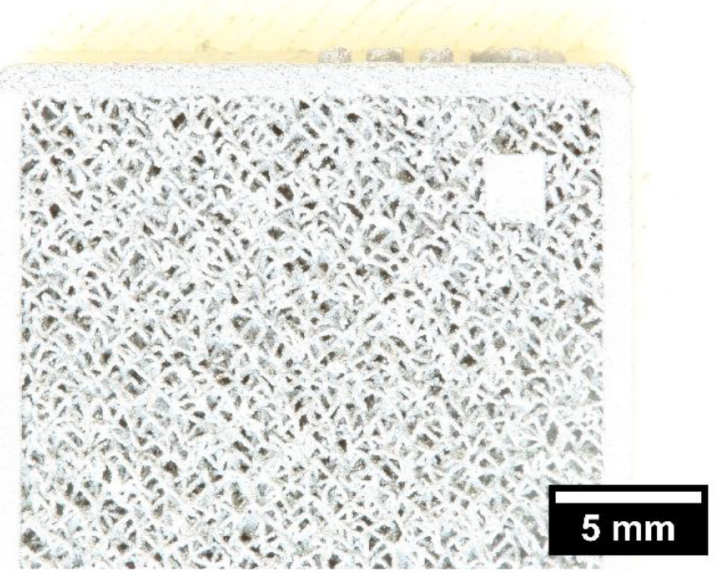

(a)

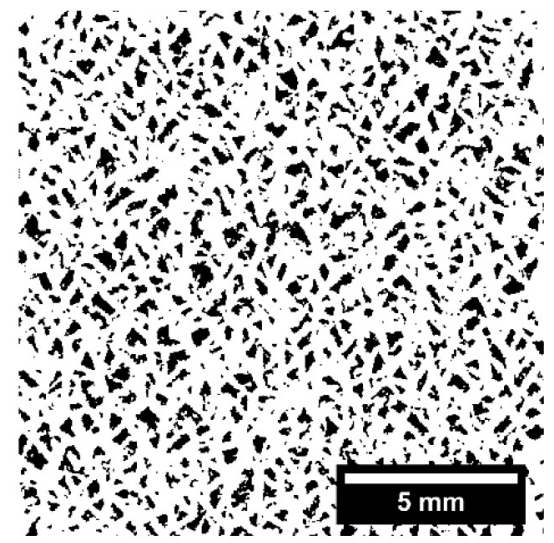

(b) 
white being the highest measured values and black the smallest) and a histogram showing the distribution of measured thicknesses was produced, as in Figure 4.

As can be seen from Figure 4, every part of the visible structure is measured using the local thickness technique described. The resulting histogram shows the distribution of the different measurement values based on the frequency with which each diameter circle occurs within the image. From this distribution, a mean value for material thickness, evaluating both strut diameter and node thickness, for each image captured of the surface of porous specimens is calculated, quantifying the entirety of the visible structure. As a result of this analysis, a single value for the solid phase of the porous structure is produced, characterising the manufactured material.

\section{Pore size analysis}

The second method of analysis used local thickness algorithms to measure the visible pore size distribution of the porous structure. By applying the local thickness analysis to the black pixels representing pores instead of the white solid material, a distribution of the pore sizes visible from the surface of the structure was produced, as shown in Figure 5.

As with local material thickness measurements, the size of every pore is determined through the fitting of circles and the frequency distribution of diameters used to calculate the mean pore size for the entire structure. Both analyses were performed in FIJI.

\section{Existing analysis methods}

To validate the image analysis method, SEM, FV and $\mu$-CT systems were used in comparison for their ability to measure material thickness and pore size. Destructive methods of porous structure analysis were not considered in this study because of their unsuitability for in-line analysis and quality assurance applications. Pore measurement methods applicable to feature sizes outside the relevant range, 45 to $1,000 \mu \mathrm{m}$, were also neglected. The SEM method, as with the photogrammetric method, requires the capture of high resolution images of the top surface of a porous structure at a magnification of $25 \times$ using a JEOL JSM-7001F field emission FEG-SEM (JEOL GmbH, DE). These images are analysed by manually drawing measurement lines across the width of the struts or pores from one edge to the other, as shown in Figure 6, to determine strut diameter and pore size. A total of 20 measurements of each geometry type were made for each sample, from which two mean values were calculated to quantify strut diameter and pore size.

The FV system, Infinite Focus SL (Alicona Imaging $\mathrm{GmbH}$, AT), unlike the DSLR and SEM-based methods, is able to capture 3D topographical data from the top surface of a porous structure (Danzl et al., 2011). From these data, the diameter of struts can be calculated, as shown in Figure 7. This is performed by fitting a cylinder to the strut and quantifying the distance above or below the nominal diameter, the mean of which was taken as the thickness of that strut. For FV measurements, a single strut was analysed in each sample to quantify the mean diameter.

$\mu$-CT data of a cylindrical porous specimen, captured using a lab-based facility (Phoenix X-ray Systems and Services $\mathrm{GmbH}$, DE) with a voxel size of $9.06 \mu \mathrm{m}$, was also used to validate measurements for pore size distribution. Pore size was analysed using accessible volume algorithms (Yue, 2011). These algorithms assessed the maximum size sphere it was possible to locate within each pore and used this to estimate the distribution of pore sizes internal to the structure (Yue, 2011). A cross-section of this analysis is shown in Figure 8.

Analysis of material thickness using all four methods described was carried out on porous structures of $600 \mu \mathrm{m}$ unit cell to replicate the trabecula bone architecture used in typical osseointegrating AM structures and match samples produced in other studies (Yue, 2011).

The results obtained for material thickness and pore size were compared with the three existing systems (SEM, FV and $\mu$-CT) to validate how accurately the image-based method matched with current methods used in industry.

Prior to testing of the analysis processes, the accuracy and minimum definable feature of the system was measured

Figure 4 (a) measurement circles fitted by local thickness analysis of a porous structure and (b) histogram of material thickness distribution

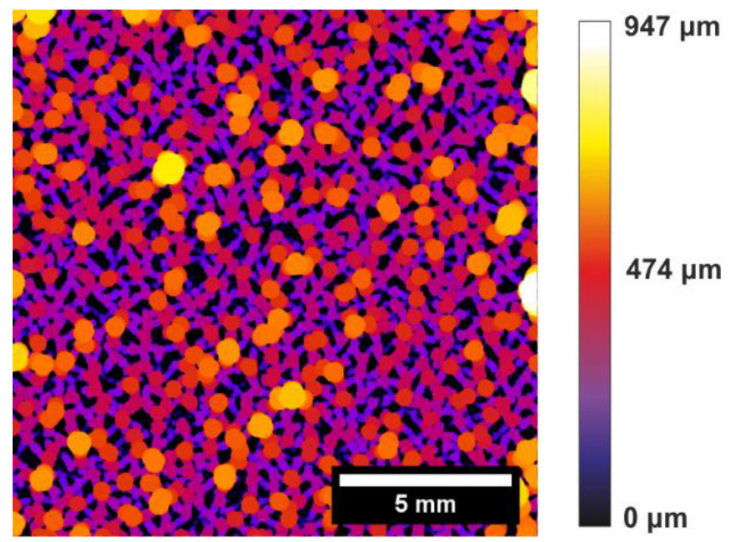

(a)

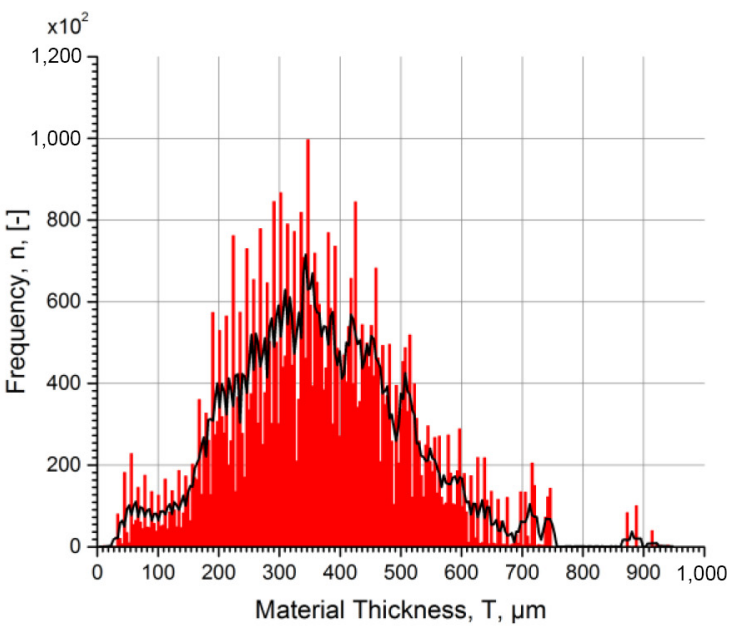

(b) 
Figure 5 (a) measurement circles fitted by local thickness analysis of porous structure and (b) histogram of pore size distribution
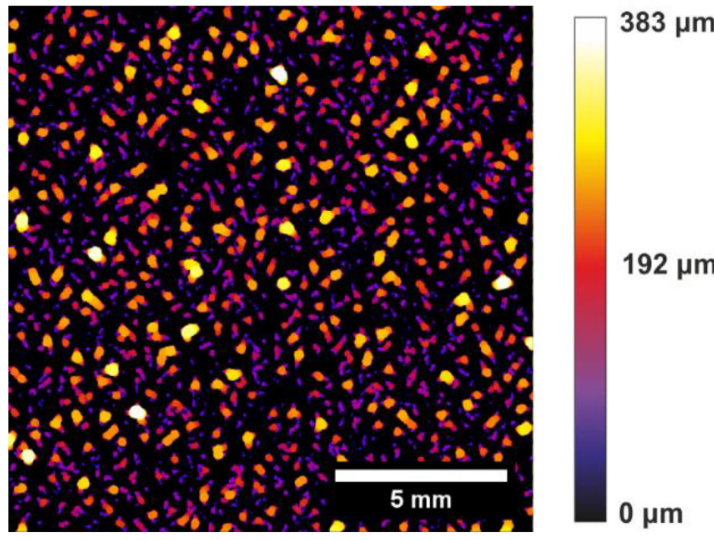

(a)

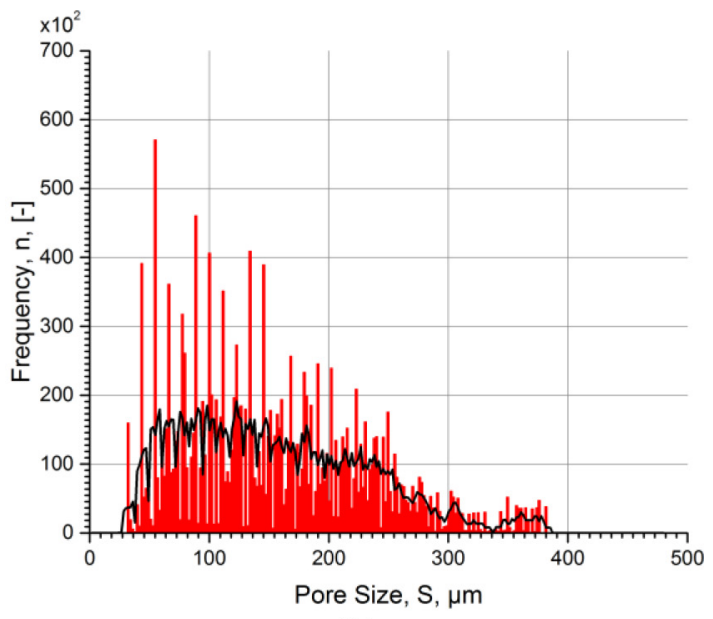

(b)
Figure 6 Output from manual placement of measurements on an SEM image to measure pore size

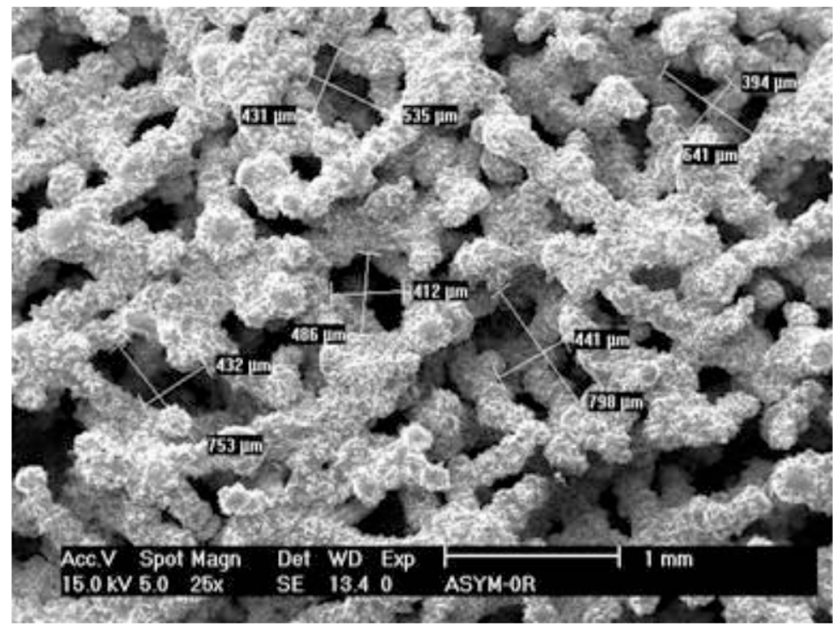

Figure 7 Output from focus variation imaging of a porous sample with a strut analysed for diameter

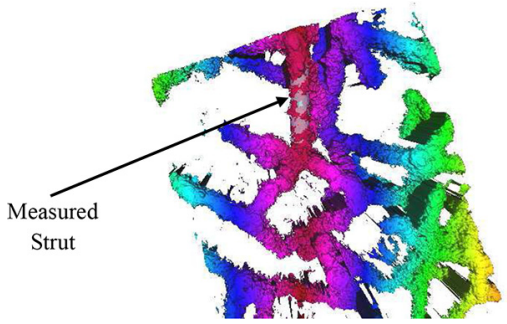

using a NBS 1952, 3" x 1", Resolution Test Target graticule (Thorlabs Inc., USA) that used simple geometries to quantify the capabilities of the camera, lens and processing methods.

Following this, analysis of a wire mesh (Locker Group Ltd., GB) of known dimensions (200 $\mu \mathrm{m}$ wire thickness, $308 \mu \mathrm{m}$ aperture) was also measured 20 times, removing and replacing the sample
Figure 8 Cross-section of spheres fitted during accessible pore size distribution analysis of a porous sample

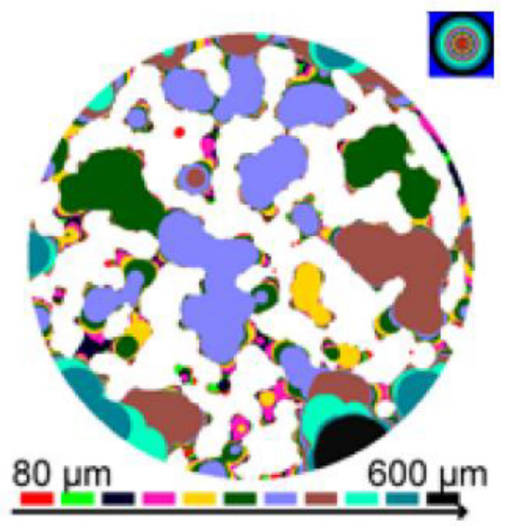

Source: Yue (2011)

between measurements, to validate consistency of the system. These measurements were used to quantify the accuracy and repeatability of the system in analysing simple 3D structures before applying the method to the more complex porous structures.

For material thickness and pore size analysis, 12 sets of measurements were taken for each of the four samples to calculate results to a margin of error of $1 \mu \mathrm{m}$, at a confidence level of 95 per cent. The required sample size was estimated using equation (7) (Mann, 2010).

$$
n=\frac{z^{2} \sigma^{2}}{E^{2}}
$$

Applying this equation to the measurements made ensured that all analysis performed by the photogrammetric system was statistically significant. The mean and standard deviation was calculated for each sample, for each method of analysis investigated, to compare to the repeatability of other measurement systems. 


\section{Results}

Validation of the photogrammetric method required four sets of analyses, each looking to test different system capabilities, specifically: resolution, repeatability, material thickness measurements and pore size measurements. Resolution analysis tested the accuracy of measurements made by the photogrammetric system and quantified the minimum measurable feature possible with the system by comparing the measured thickness of line sets on the target test graticule to the stated values. Repeatability analysis investigated whether the system could repeatedly determine the thickness of a wire mesh of known dimensions and assess the system's ability to measure 3D surfaces, and not only printed slides. Material thickness analysis was performed to determine the measured value produced by the system and how these were compared to values acquired for SEM and FV. Finally, pore size analysis was investigated to determine how accurately results from imaging were compared to data obtained from SEM analysis and $\mu-\mathrm{CT}$ data analysed via accessible pore size.

\section{System resolution analysis}

Resolution and accuracy of the analysis system were tested using a resolution test target graticule. This graticule, composing of a number of groups of vertical and horizontal lines with various widths and spacing, allowed for the minimum resolvable feature size of the system and the measurement accuracy to be quantified. Images were captured and processed identically to the porous specimens in the study, as shown in Figure 9.

As can be seen in Figure 9, small features within the resolution graticule can be distorted following image processing, as a result of the resolution limits of the imaging system and noise, rounding measurements between the pixel size of $8 \mu \mathrm{m}$, up or down. This limitation, however, only affected measurement lines below a thickness of $18 \mu \mathrm{m}$.

To determine the values measured for each set of lines by the system, local thickness analysis was applied to the processed image to create a corresponding histogram. The outcome of the local thickness analysis along with the actual and predicted line width histogram is shown in Figure 10.

The width measurements of the line sets of the resolution test graticule, calculated via local thickness analysis, were compared to the quoted values, and the accuracy of measurements determined as shown in Table I. Values measured for the graticule were taken from the peaks observed in the histogram of measurement values and aligned with the closest expected

Figure 9 Processed image of a resolution test graticule following cropping, conversion to 8-bit greyscale, thresholding and filtering

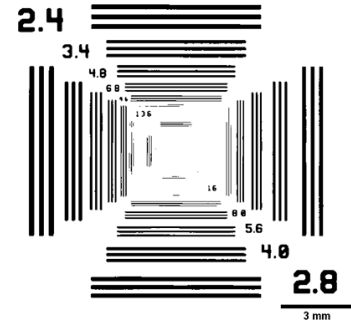

line width to determine the error in measurement of the imaging system.

Analysis of images captured of the resolution test target showed that the system was able to detect and accurately measure a wide range of line groups on the graticule. Specifically, line widths between 208 and $63 \mu \mathrm{m}$ were measured at or close to the expected value. As the difference between line thickness values dropped below the pixel resolution limit of the system, it became more difficult to distinguish different thicknesses, causing measurements to be grouped with a higher or lower value. The minimum resolvable feature measured was $18 \mu \mathrm{m}$, equivalent to a resolution of 28 lines per $\mathrm{mm}$, well below the minimum thickness of a strut within a porous structure or the average powder diameter (Thorlabs Inc., 2016). These measurements show that the imaging and analysis method is able to detect and quantify simple geometries of known size down to a value suitable for measuring open cell porous orthopaedic surfaces. Analysis of the results quantifies the error in measurements at $2.9 \mu \mathrm{m}$ down to a line width of $18 \mu \mathrm{m}$, demonstrating high accuracy across a wide range of measurement values.

\section{Wire mesh analysis}

To validate the capability of the system to measure $3 \mathrm{D}$ porous materials, a simple mesh of known size was analysed. Images of the mesh were captured and processed in an identical manner to the resolution test target and subsequent porous material, as shown in Figure 11, to determine values for wire thickness (Figure 12) and aperture size (areas between wire strands, as in Figure 13).

The values of wire diameter and aperture size, as measured by the local thickness algorithms, were compared to the expected result to determine accuracy of the system and the level of variation determined, as shown in Table II.

The photogrammetric system over-measured the thickness of the mesh wire by 13 per cent of the expected value. This over-measurement is partially a result of the limited resolution of the system but is also caused by the geometry of the mesh. Although the wire of the mesh is $200 \mu \mathrm{m}$, the areas where the wire crosses over are wider diagonally $(282 \mu \mathrm{m})$, which is detected by the local thickness analysis as seen in the analysis histogram in Figures 12 and 14.

The two peaks have mean value of 195 and $267 \mu \mathrm{m}$, respectively, 5 and $16 \mu \mathrm{m}$ from the $200 \mu \mathrm{m}$ and $282 \mu \mathrm{m}$ known geometry values of the mesh. This causes overmeasurement of the mesh thickness compared to the expected value; however, the existence of the two peaks, and the high accuracy with which the image-based technique quantifies the diameter of the wire compared with known values, demonstrates an ability to accurately measure simple 3D structures. As with test graticule measurements, resolution limits also caused small amounts of deviation from the expected value for both material thickness and aperture size, with the latter varying by 6 per cent of the $308 \mu \mathrm{m}$ expected value. While able to measure close to the expected values, the photogrammetric system also demonstrated a very low deviation, even after repeated measurements, of $3.9 \mu \mathrm{m}$ and $1.8 \mu \mathrm{m}$ for material thickness and aperture size, respectively, indicating high repeatability of the technique even with deliberate removal and replacement of the test specimen. 
Figure 10 (a) Measurement circles fitted by local thickness analysis of the resolution test graticule and (b) histogram of expected and measured line widths, and minimum pixel resolution of the imaging apparatus

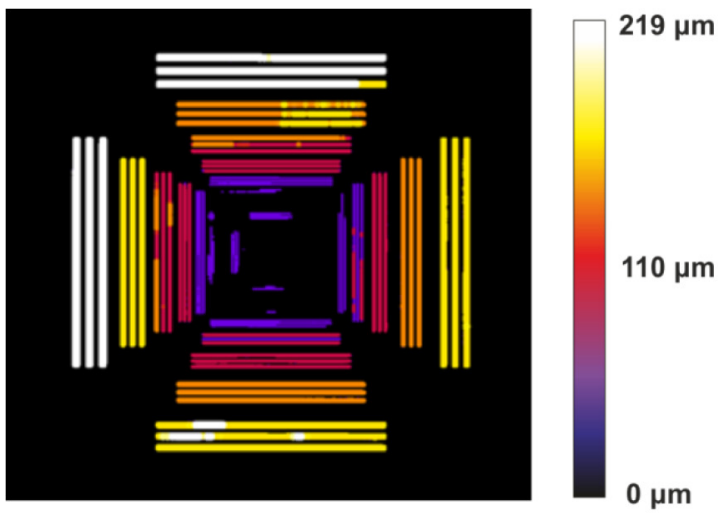

(a)

Source: Thorlabs Inc. (2016)

Table I Comparison between expected and measured values of resolution test graticule, all line widths below $18 \mu \mathrm{m}$ were not distinguished

\begin{tabular}{lcc}
\hline $\begin{array}{l}\text { Expected line width, } T_{E,} \\
\mu \mathrm{m}\end{array}$ & $\begin{array}{c}\text { Measured line width, } T_{M,}, \\
\mu \mathrm{m}\end{array}$ & $\begin{array}{c}\text { Difference, } \Delta T, \\
\mu \mathrm{m}\end{array}$ \\
\hline 208 & 208 & 0 \\
177 & 174 & 3 \\
147 & 151 & 4 \\
124 & 127 & 3 \\
103 & 104 & 1 \\
89 & 92 & 3 \\
74 & 69 & 5 \\
63 & 58 & 5 \\
52 & Not distinguished & - \\
45 & 46 & 1 \\
42 & Not distinguished & - \\
37 & 35 & 2 \\
31 & Not distinguished & - \\
29 & Not distinguished & - \\
25 & Not distinguished & - \\
21 & 23 & 2 \\
18 & 12 & 6 \\
Source: Thorlabs Inc., 2016 & & \\
\hline
\end{tabular}

These measurements, and the subsequent analysis, therefore indicate the system to be suitable for algorithmically measuring metallic open cell structures at high accuracy and in a consistent manner from captured images.

Material thickness measurements

Having been validated for the measurement of simple structures of known dimension, the system was investigated for its ability to measure the thickness of material comprising the porous structure. Initial analysis of the local thickness data taken from measurements showed that the mean value calculated for the material thickness was skewed by the large

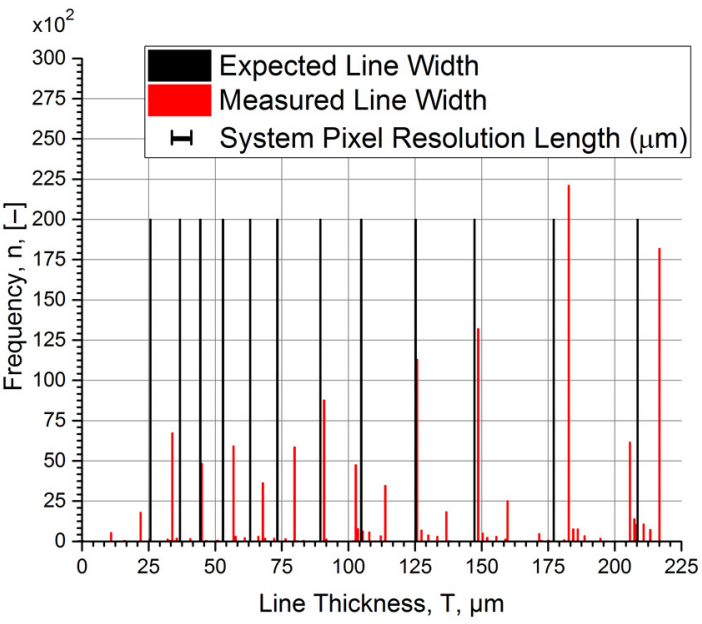

(b)

Figure 11 Processed image of a wire mesh

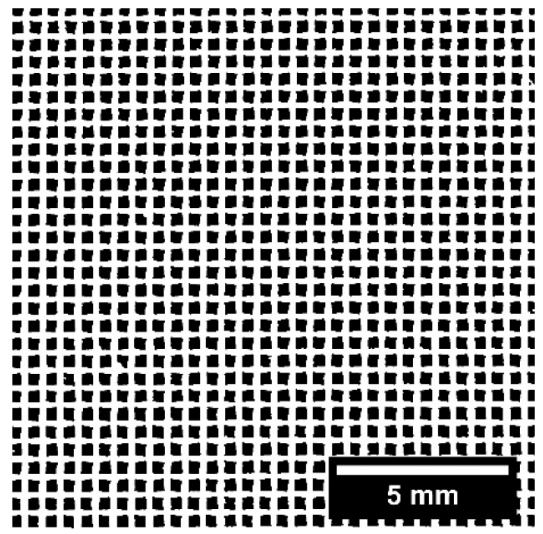

Figure 12 Local thickness analysis of the mesh wire

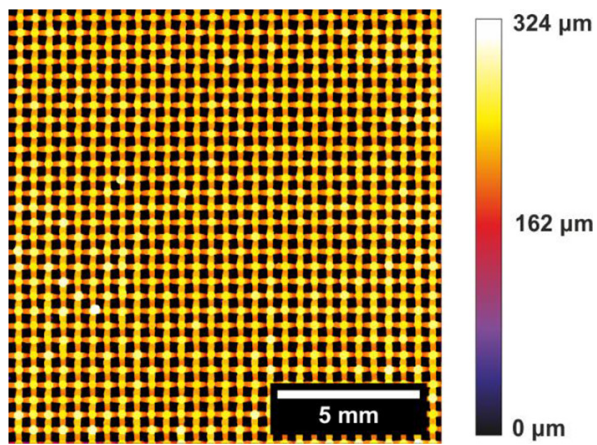

amount of " $0 \mu \mathrm{m}$ " values produced by thickness measurements of black pixels in the image, despite them having no value. To account for this, the $0-\mu \mathrm{m}$ peak was removed from the data before calculating the mean of the histogram distribution. The four porous specimens used were also analysed using both the SEM and FV imaging methods. The 
Figure 13 Local thickness analysis of mesh apertures

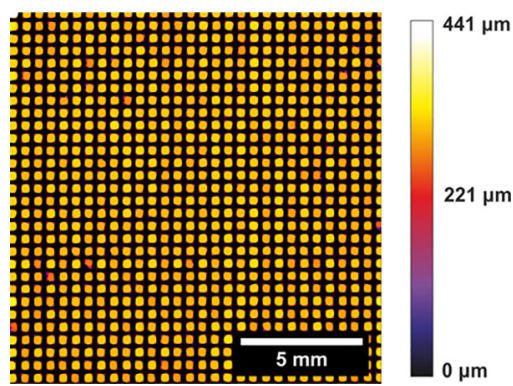

Table II Material thickness and aperture size measurements of a wire mesh obtained from photogrammetric analysis

\begin{tabular}{lccc}
\hline $\begin{array}{l}\text { Measurement } \\
\text { type }\end{array}$ & $\begin{array}{c}\text { Thickness, T, } \\
\mu \mathrm{m}\end{array}$ & $\begin{array}{c}\text { Standard deviation, } \\
\sigma, \mu \mathrm{m}\end{array}$ & $\begin{array}{c}\text { Difference, } \Delta T \text {, } \\
\mu \mathrm{m}\end{array}$ \\
\hline Material & & & \\
thickness & 226 & 3.9 & 26 \\
Aperture size & 290 & 1.8 & 18 \\
\hline
\end{tabular}

Figure 14 Histogram of photogrammetric mesh wire measurements showing expected results at $200 \mu \mathrm{m}$ and $282 \mu \mathrm{m}$

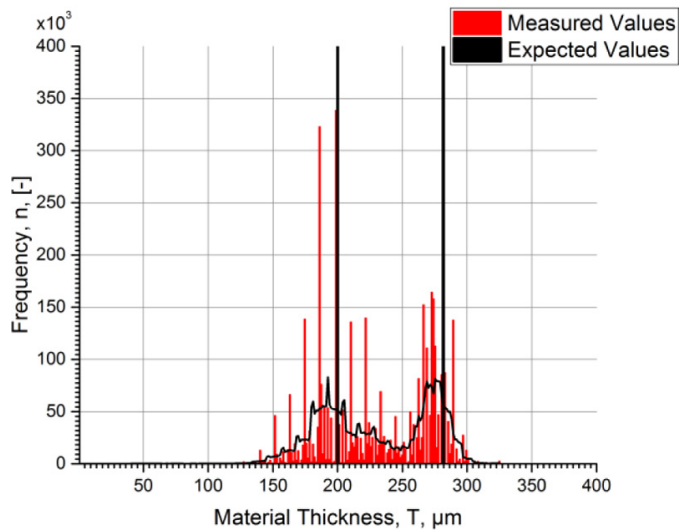

results for each analysis method used in determining strut diameter are shown in Table III. A total of 12 images were captured and analysed for each sample with the photogrammetric system, removing and replacing the component each time, determined by equation (7).

Table III shows the measurements taken for material thickness for each of the three applicable methods across the four test specimens. The majority of material thickness measurements taken by the SEM and FV systems aligned with the expected strut diameter of 180 to $220 \mu \mathrm{m}$ range, whereas those made by the photogrammetric system were measured above this range, between 412 and $431 \mu \mathrm{m}$, primarily due to the inclusion of nodes within the analysis that possessed wider diameters, as with the more simplistic wire mesh. Measurements made by the photogrammetric system however showed the smallest range and a lower standard deviation than that of SEM method. This low standard deviation
Table III Material thickness measurements obtained from photogrammetric, SEM and FV analysis of four porous samples

\begin{tabular}{lccc}
\hline $\begin{array}{l}\text { Measurement } \\
\text { method }\end{array}$ & $\begin{array}{c}\text { Sample } \\
\text { No. }\end{array}$ & $\begin{array}{c}\text { Mean material } \\
\text { thickness, } \mathbf{T}, \mu \mathbf{m}\end{array}$ & $\begin{array}{c}\text { Standard } \\
\text { deviation, } \sigma, \mu \mathbf{m}\end{array}$ \\
\hline Photogrammetric & 1 & 417 & 9 \\
& 2 & 412 & 7 \\
& 3 & 428 & 5 \\
SEM & 4 & 431 & 6 \\
& 1 & 206 & 12 \\
Focus variation & 2 & 178 & 15 \\
& 3 & 220 & 25 \\
& 2 & 219 & 21 \\
& 2 & 184 & No data \\
& 3 & 185 & No data \\
& 4 & 221 & No data \\
\hline
\end{tabular}

demonstrates the system is able to measure porous structures consistently and with high repeatability. Specific measurement values also followed similar trends for all three measurement methods, with Samples 1 and 2 shown to have lower thickness than Samples 3 and 4.

\section{Pore size comparison}

Of the systems used in analysing the porous material, only the imaging, SEM and $\mu$-CT systems were capable of measuring pore size distribution. Similar to material thickness measurements, local thickness analysis was used to measure the width of the visible pores in a $600-\mu \mathrm{m}$ unit cell structure. The values measured by the imaging system are shown in Table IV.

Measurements made using the image-based system showed a mean pore size of $158 \mu \mathrm{m}$ with a deviation of 3 $\mu \mathrm{m}$ between the four samples analysed. Measurements for mean pore size using the SEM based line drawing method showed the average pore size to be $450 \mu \mathrm{m}$ with a standard deviation of $110 \mu \mathrm{m}$. Analysis of a sample using $\mu-\mathrm{CT}$ scanning determined the modal value of pore size to be 324 $\mu \mathrm{m}$ (Yue, 2011). As with material thickness, while measurements made using the three methods disagreed, the photogrammetric system was shown to produce a highly repeatable analysis.

\section{Discussion}

Resolution testing showed the system was capable of accurately measuring simple geometric shapes and distinguishing features down to a size of $63 \mu \mathrm{m}$, producing results with an accuracy as

Table IV Average pore size measurements obtained from photogrammetric analysis of four porous samples, SEM analysis and $\mu$-CT analysis

\begin{tabular}{lcc}
\hline Measurement method & Mean pore size, S, $\mu \mathbf{m}$ & $\begin{array}{c}\text { Standard deviation, } \\
\sigma, \mu \mathbf{m}\end{array}$ \\
\hline Photogrammetric & 158 & 2 \\
SEM & 450 & 110 \\
$\mu-\mathrm{CT}$ & 324 & No data \\
\hline
\end{tabular}


low as $1 \mu \mathrm{m}$ up to a maximum of $12 \mu \mathrm{m}$. The system was able to measure lines below the $63 \mu \mathrm{m}$ value however, due to the difference in width between line groups being less than the resolution of the camera these lines were not always differentiated from one another. Despite the grouping of similar lines, the system was still able to accurately measure values required for the analysis of porous material, down to a size of $18 \mu \mathrm{m}$ with a mean error of $2.9 \mu \mathrm{m}$, demonstrating the system to be accurate and able to measure a small enough feature for analysing orthopaedic porous material, limited only by the minimum resolution of the system. The results acquired show the precision of the hardware used in the system to be suitable for the required application of quality control and inspection at the scale needed for $\mathrm{AM}$ porous structures, following further in-situ validation.

Measurement of the wire mesh showed the system was able to produce measurements for both wire and aperture size. Although some inaccuracies were observed in measuring the wire (13 per cent over measurement for $200 \mu \mathrm{m}$ wire thickness and 6 per cent under measurement for $308 \mu \mathrm{m}$ aperture size), particularly where two strands overlapped producing a larger observable area, the system produced very consistent measurement values with low deviations of $3.9 \mu \mathrm{m}$ and $1.8 \mu \mathrm{m}$ for wire thickness and aperture size respectively, even when removing and replacing the test specimen before every image capture. These measurements validated the developed system's ability to consistently and accurately measure simple material geometries from images captured at the surface prior to investigation of more complex orthopaedic structures. Analysis of the mesh additionally showed the system to reject deliberate variation through removing and replacing the specimen between images while maintaining a low standard deviation of measurements.

The photogrammetric system produced material thickness measurements of the porous material, up to $431 \mu \mathrm{m}$. This compared highly to the $220 \mu \mathrm{m}$ and $252 \mu \mathrm{m}$ for the SEM and FV methods, respectively. The primary cause of these higher measurements was the photogrammetric systems capability of measuring the entirety of the porous structure, both struts and nodes. While simple geometric shapes such as those in the resolution test graticule and wire mesh were measured with high accuracy, randomised structures were further from the predicted range as a result of overlapping struts at different depths and nodes at the connection of struts. Nodes are larger in diameter than the struts, thus inflating the value of the mean measured size. Because of the random nature and complexity of the structure and range of sizes measured, removal of the nodes would be difficult, potentially time consuming and would mean the system no longer analysed the material completely. Despite over measurement, photogrammetric analysis was shown to have a low deviation compared to that of the SEM measurements due primarily to the automated, algorithmic nature of the analysis, contrary to the manual measurements used by the SEM process which is inherently unrepeatable. The repeatable nature of measurements and the correlation between samples of higher or lower material thickness across all three measurements indicate the photogrammetric system's ability to successfully analyse AM porous components. By producing measurements of the same porous material at lower deviation than existing methods while carrying out rapid and low complexity analysis, the imagebased system is shown to meet the requirements for in-line inspection of large volumes of manufactured parts that was previously impractical.

Measurements of pore size also varied between the photogrammetric, SEM and $\mu$-CT systems. The imagebased technique measured the lowest value for pore diameter with a mean of $158 \mu \mathrm{m}$. SEM measurements were higher, at $450 \mu \mathrm{m}$. Finally the $\mu$-CT system determined pore size to be $324 \mu \mathrm{m}$. The cause of the discrepancy between the systems is primarily due to the measuring of different parts of the pores. As a result of using 3D data, $\mu$-CT analysis measures the pores most completely, using the generated model to determine where the edges of the internal pores lay. Conversely, SEM and photogrammetric analysis is only capable of 2D measurements from the surface of a sample, reducing how completely the pore size is measured. SEM and image analysis also differed from one another due to how the edges of pores are detected. While SEM requires edges of pores to be located manually, image analysis uses the black space between the struts to determine where a pore edge lies, meaning only areas where no visible material exists are taken to be pores, even if the actual pore is larger. While this creates inaccuracies when attempting to determine the pore size of a material, this type of analysis is still beneficial for quality control applications due to the repeatable nature of the AM process and image analysis methodology when fabricating and inspecting porous structures. Each pore is built with the same size and location within the structure of a component and then measured using the same method each time, making it possible to track variation in pore size using measurement data previously acquired. Even with disagreement between the three systems. Pore size measurements by the imagebased system were shown to be highly repeatable, with a deviation of $2 \mu \mathrm{m}$ compared with that of the existing SEM method of $110 \mu \mathrm{m}$, a product of measuring both the width and length of highly irregular shaped pores.

The high repeatability seen in material thickness and pore size measurements suggest the photogrammetric method is well suited for application of process and variation monitoring, where detecting changes in the built structure with precision is paramount to measurement accuracy as long as images are captured consistently. The mean material thickness of the porous structure, determined from the analysis described previously, possesses the necessary functionality to track uncontrollable changes to part properties, resulting from variation in the $\mathrm{AM}$ build conditions. This comparative application for measured values from the photogrammetric system also implies that the negative attributes of analysing a $3 \mathrm{D}$ structure using $2 \mathrm{D}$ images i.e. line of sight and depth of measurement issues can be neglected as long as images of the porous structures are captured in a repeatable manner at the same location for each specimen, as demonstrated in this paper. The four analyses performed each demonstrate step-by-step validation of the newly developed imaging system and its suitability for quantifying $\mathrm{AM}$ porous components precisely and repeatedly, for both solid material and open cell pores. 


\section{Conclusions}

A low-cost, rapid method of analysing porous open cell structures, manufactured via laser-based powder bed AM, has been demonstrated. This method is capable of capturing images of porous structures and quantifying properties of the material. Quantitative analysis of the techniques used indicates the system is able to measure simple geometric shapes in the form of resolution test lines and simple wire meshes. High accuracy was observed with the system able to measure down to a minimum feature size of $18 \mu \mathrm{m}$, although line groups closer than the resolution of the system were grouped together due to limits of the hardware. A wire mesh of known dimensions was used to quantify the repeatability of the method when measuring simple geometric shapes. Analysis showed the system to be capable of measuring close to the expected values, with the geometry of the mesh causing some over measurement of wire strands and the resolution of the system showing small under measurements of the mesh apertures. The system showed a very low deviation across repeated measurements indicating a very high consistency and suitability for variation tracking and comparative go/no go inspection.

The system was also capable of analysing material thickness and pore size of randomised porous material, although results obtained differ when compared to those measured by $\mu$-CT, FV and SEM methods with material thickness measurements between the SEM, FV and photogrammetric system almost double at the most extreme. One cause of this is the additional measurement of nodes alongside struts within the porous material. While the other systems only measured strut diameters, photogrammetric analysis also measured thicker nodes that increased the value for mean material thickness. Disagreement was also seen in measurements made of pore size. The primary cause of this discrepancy was due to the different ways in which each system selected the edges of pores when taking measurements. Despite this disparity, the repeatable nature of the AM process and the image based analysis was demonstrated with both material thickness and pore size showing lower standard deviations across many measurements compared to existing methods. This validated the photogrammetric systems suitability for analysing porous components, indicating the occurrence of variation during the manufacture of porous structures. Further validation of this system in an industrial context would determine the suitability for applications in process quality control and assurance.

Qualitatively the system exhibits some advantages over other methods studied. Aside from the low cost and reduced analysis time, the system is also capable of measuring larger areas of a sample than SEM, FV or $\mu$-CT. This allows for a more accurate calculation of mean material thickness and pore size without the need for repeated sampling. The algorithmic based analysis has high automation, reducing potential for error caused by manual operation. Unlike other systems the image based analysis requires less specialised knowledge to operate as only experience in commercial camera operation and of the accompanying software is required. The benefits of this method lends the system to quality control applications requiring inspection of a high volume of components and to the tracking of process variability caused by changes in parameters and environmental conditions within AM manufacturing. The high speed, repeatability and consistency of the photogrammetric system show it to be better suited for tracking of variation in manufacturing processes, which cause changes in the material thickness of the entire imaged porous structure, than for absolute measurements of individual pores and struts due to the complete measurement of a large sample area of the irregular 3D structure unlike other methods that primarily target specific struts or pores.

\section{References}

Bobyn, J.D., Toh, K., Hacking, S.A., Tanzer, M. and Krygier, J.J. (1999), "Tissue response to porous tantalum acetabular cups: a canine model”, The Fournal of Arthroplasty, Vol. 14 No. 3, pp. 347-354.

Canon Inc (2015), "Canon EOS 70D instruction manual", [online], available at: www.canon.co.uk/ (Accessed 08 June 2016).

Danzl, R., Helmli, F. and Scherer, S. (2011), "Focus variation-a robust technology for high resolution optical 3D surface metrology", Strojniški Vestnik - Fournal of Mechanical Engineering, Vol. 2011 No. 3, pp. 245-256.

Dougherty, R. and Kunzelmann, K.H. (2007), "Computing local thickness of 3D structures with ImageJ", Microscopy and Microanalysis, Vol. 13(SupplementS02), pp. 1678-1679. Supp1S02).

Friedman, R.J., Black, J., Galante, J.O., Jacobs, J.J. and Skinner, H.B. (1993), "Current concepts in orthopaedic biomaterials and implant fixation", fournal of Bone and foint Surgery-American, Vol. 75 No. 7, pp. 1086-1109.

Hildebrand, T. and Rüegsegger, P. (1997), "A new method for the Model-Independent assessment of thickness in ThreeDimensional images", fournal of Microscopy, Vol. 185 No. 1, pp. 67-75.

Gonzalez, R.C. and Woods, R.E. (2008), Digital Image Processing, Pearson/Prentice Hall.

Jeyapalina, S., Beck, J.P., Bloebaum, R.D. and Bachus, K.N. (2014), "Progression of bone ingrowth and attachment strength for stability of percutaneous osseointegrated prostheses",

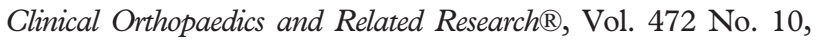
pp. 2957-2965.

Leach, R. (2011), Optical Measurement of Surface Topography, Springer, Berlin Heidelberg.

Mann, P.S. (2010), Introductory Statistics, John Wiley \& Sons, Hoboken, NJ, US.

Mullen, L., Stamp, R.C., Brooks, W.K., Sutcliffe, C.J. and Jones, E. (2009), "Selective laser melting: a regular unit cell approach for the manufacture of porous, titanium, bone inGrowth Constructs, suitable for orthopedic applications", Fournal of Biomedical Materials Research Part B: Applied Biomaterials, Vol. 89B No. 2, pp. 325-334.

Mullen, L., Stamp, R.C., Fox, P., Jones, E., Ngo, C. and Sutcliffe, C.J. (2010), "Selective laser melting: a unit cell approach for the manufacture of porous, titanium, bone in-Growth Constructs, suitable for orthopedic applications. II. Randomized structures", fournal of 
Biomedical Materials Research Part B: Applied Biomaterials, Vol. 92B No. 1, pp. 178-188.

Muth, J., Poggie, M., Kulesha, G. and Meneghini, R.M. (2013), "Novel highly porous metal technology in artificial hip and knee replacement: processing methodologies and clinical applications", The fournal of the Minerals, Metals $\mathcal{E}$ Materials Society, Vol. 65 No. 2, pp. 318-325.

Robinson, J. (2014), Optimisation of the Selective Laser Melting Process for the Production of Hybrid Orthopaedic Devices, The University of Liverpool, Liverpool, UK. [Unpublished].

Ryan, G., Pandit, A. and Apatsidis, D.P. (2006), "Fabrication methods of porous metals for use in orthopaedic applications", Biomaterials, Vol. 27 No. 13, pp. 2651-2670.

Schindelin, J., Arganda-Carreras, I., Frise, E., et al. (2012), "Fiji: an open-source platform for biological-image analysis", Nature Methods, Vol. 9 No. 7, pp. 676-682.PMID 22743772, doi:10.1038/nmeth.2019.
Steen, W., Watkins, K.G. and Mazumder, J. (2010), Laser Material Processing, Springer, London.

Thorlabs Inc (2016), "Resolution test targets", [online] available at: www.thorlabs.com/newgrouppage9.cfm?objectgroup_id= 4338 (Accessed 11 August 2016).

Tomlins, P., Grant, P., Mikhalovsky, S., James, S. and Mikhalovska, L. (2004), "Measurement of pore size and porosity of tissue scaffolds", Fournal of ASTM International, Vol. 1 No. 1, pp. 1-8.

Vanderesse, N., Ky, I., Quevedo González, F., Nuño, N. and Bocher, P. (2016), "Image analysis characterization of periodic porous materials produced by additive manufacturing", , Vol. 92, pp. 767-778.

Yue, S. (2011), "Non-Destructive Quantification of Tissue Scaffolds and Augmentation Implants Using X-Ray Microtomography”, Imperial College London, London, UK.

\section{Corresponding author}

Samuel Evans can be contacted at: evanss@liverpool.ac.uk 